\title{
Achieving Cost-efficient, Data-intensive Computing in the Cloud
}

\author{
Michael Conley \\ University of California, San Diego \\ mconley@cs.ucsd.edu
}

\author{
Amin Vahdat \\ Google Inc. \\ University of California, San Diego \\ vahdat@cs.ucsd.edu
}

\author{
George Porter \\ University of California, San Diego \\ gmporter@cs.ucsd.edu
}

\begin{abstract}
Cloud computing providers have recently begun to offer high-performance virtualized flash storage and virtualized network I/O capabilities, which have the potential to increase application performance. Since users pay for only the resources they use, these new resources have the potential to lower overall cost. Yet achieving low cost requires choosing the right mixture of resources, which is only possible if their performance and scaling behavior is known.

In this paper, we present a systematic measurement of recently introduced virtualized storage and network I/O within Amazon Web Services (AWS). Our experience shows that there are scaling limitations in clusters relying on these new features. As a result, provisioning for a large-scale cluster differs substantially from small-scale deployments. We describe the implications of this observation for achieving efficiency in large-scale cloud deployments. To confirm the value of our methodology, we deploy cost-efficient, highperformance sorting of $100 \mathrm{~TB}$ as a large-scale evaluation.
\end{abstract}

Categories and Subject Descriptors C.4 [Performance of Systems]: Measurement techniques

General Terms Performance, Measurement

Keywords Cloud, I/O performance

\section{Introduction}

Cloud providers such as Amazon Web Services (AWS) [3], Google Cloud Platform [9] and Microsoft Azure [4] offer nearly instantaneous access to configurable compute and storage resources that can grow and shrink in response to application demands, making them ideal for supporting largescale data processing tasks. Yet supporting the demands of

Permission to make digital or hard copies of all or part of this work for personal or classroom use is granted without fee provided that copies are not made or distributed for profit or commercial advantage and that copies bear this notice and the full citation on the first page. Copyrights for components of this work owned by others than ACM must be honored. Abstracting with credit is permitted. To copy otherwise, or republish, to post on servers or to redistribute to lists, requires prior specific permission and/or a fee. Request permissions from permissions@acm.org.

SoCC '15, August 27-29, 2015, Kohala Coast, HI, USA

C2015 ACM. ISBN 978-1-4503-3651-2/15/08\$15.00

DOI: http://dx.doi.org/10.1145/2806777.2806781 modern Internet sites requires not just raw scalability, but also cost- and resource-efficient operation: it is critical to minimize the resource budget necessary to complete a particular amount of work, or conversely to maximize the amount of work possible given a particular resource budget.

Minimizing cloud costs requires choosing a combination of resources tailored to a given application and workload. There have been several measurement studies of the performance of cloud resources [17, 20, 32], and several efforts aimed at automatically selecting a configuration of cloud resources suited to a given workload [14, 15, 34]. This is no easy task, as the diversity within public cloud platforms has rapidly accelerated over the past half decade. For example, as of this writing, Amazon offers 53 different types of VMs, differing in the number of virtual CPU cores, the amount of memory, the type and number of local storage devices, the availability of GPU processors, and the available bandwidth to other VMs in the cluster. The above-mentioned provisioning tools have shown promise, especially for resources such as CPU time and memory space, which can be precisely divided across tenant VMs located on the same hypervisor. On the other hand, shared resources, such as network bandwidth and storage, have proven to be a bigger challenge [10,33].

Recent advances in virtualized I/O, such as SR-IOV [28], have the potential to improve efficiency by allowing virtualized access to fast solid-state drives and high speed networking. Because users pay only for the resources they use, greater efficiency leads to lower costs. However, choosing the right set of resources in this environment is harder than ever, given that the configuration space is now even larger than before. Further, as the size of the cluster increases, overall cluster utilization and efficiency can drop, requiring more VMs to meet performance targets and driving up overall cost [7]. Thus an understanding of the scaling behavior of virtualized cloud network and storage resources is key to achieving cost-efficiency in any large deployment.

In this paper, we describe a systematic measurement of the scaling properties of recently-introduced virtualized network and storage resources within the AWS public cloud. Our aim is to determine the optimal price points for configuring clusters for data-intensive applications, specifically applications that are I/O-bound. We deploy Themis [22], our 
in-house implementation of MapReduce, as a case study of I/O-bound data processing applications under a variety of efficiency and data durability assumptions. We present a largescale evaluation of our methodology using jobs drawn from the annual 100 TB "GraySort" sorting competition [27].

We find that despite newly-introduced I/O virtualization functionality, AWS clusters still have scalability limitations, leading to larger cluster sizes than would be otherwise predicted from the performance of small numbers of nodes. We further find that the choice of cloud resources at scale differs significantly from predicted configurations measured at smaller scale. Thus the actual deployment cost shifts dramatically from estimates based on small-scale tests.

We further show that, by measuring performance at scale, it is possible to provision highly efficient clusters in the AWS public cloud. As a demonstration of this point, we deploy Themis MapReduce to an AWS cluster consisting of 100s of nodes and 100s of terabytes of virtualized SSD storage, and set three new world records in the GraySort competition at very low cost. We compare our sorting results to other record winners, and find several commonalities between the winning entries, further supporting the results of this work.

The contributions of this paper are:

1. A systematic methodology for measuring the I/O capabilities of high-performance VMs in the public cloud via application-level benchmarks.

2. A measurement of the current AWS offerings at scale, focusing on virtualized I/O.

3. A large-scale evaluation of cost-efficient sorting on 100s of nodes and 100s of terabytes of data informed by this measurement methodology.

4. Three new world records in sorting speed and costefficiency based on our evaluation results.

\section{Related Work}

While many previous works have studied performance in the public cloud, we note that our work is unique in that it has the following aspects:

- We measure clusters composed of 100s of VMs

- We measure VMs offering high-performance virtualized storage and network devices

- We measure workloads making use of 100 s of terabytes of cloud-based storage

- We use the results of our study to break several world records in high-speed sorting.

We now discuss several related studies.

Measurement: Many have measured the public cloud's potential as a platform for scientific computing. Walker [32] compared Amazon Elastic Compute Cloud (EC2) to a state-of-the-art high-performance computing (HPC) cluster. Mehrotra et al. [20] performed a similar study four years later with NASA HPC workloads. Both came to the same

\begin{tabular}{l|r|r|r|r} 
Type & vCPU & RAM & Storage & Net. \\
\hline $\mathrm{m} 1$. small & 1 & $1.7 \mathrm{~GB}$ & $160 \mathrm{~GB}$ & Low \\
$\mathrm{m} 3 . x$ large & 4 & $15 \mathrm{~GB}$ & $80 \mathrm{~GB}^{\star}$ & High \\
hs1.8xlarge & 16 & $117 \mathrm{~GB}$ & $49 \mathrm{~TB}$ & $10 \mathrm{G}$ \\
i2.8xlarge & 32 & $244 \mathrm{~GB}$ & $6.4 \mathrm{~TB}^{\star}$ & $10 \mathrm{G}$
\end{tabular}

Table 1: Four EC2 VMs with various CPU, memory, storage, and network capabilities. Some VMs use flash $\left(^{\star}\right)$ rather than disk.

conclusion that the network in the public cloud simply is not fast enough for HPC workloads.

Others have have studied the impact of virtualization on I/O resources. Wang and $\mathrm{Ng}$ [33] measure a wide variety of networking performance metrics on $\mathrm{EC} 2$ and find significantly more variance in $\mathrm{EC} 2$ than in a privately owned cluster. Ghoshal et al. [10] study storage I/O and find that EC2 VMs have lower performance and higher variability than a private cloud designed for scientific computation.

Variability in the cloud extends to CPU and memory resources as well. Schad et al. [25] measure the variability of a wide variety of VM resources and find that among other things, heterogeneity in the underlying server hardware dramatically increases performance variance. Two VMs of the same type may run on different processor generations with different performance profiles.

In a somewhat different line of study, Li et al. [17] measure inter-cloud variance, that is, the difference in performance between cloud providers. They compare Amazon EC2, Microsoft Azure, Google AppEngine and RackSpace CloudServers across a variety of dimensions and find that each cloud provider has its own performance profile that is substantially different from the others, further complicating the choice of resource configuration in the public cloud.

Configuration: One goal of measuring the cloud is optimal, automatic cluster configuration. Herodotou et al. [14] describe Elasticizer, a system that profiles Hadoop MapReduce jobs and picks an optimal job configuration on EC2. Wieder et al. [34] present Conductor, a system that combines cloud services and local servers in a single deployment.

Scheduling around deadlines in shared clusters is another common line of work. ARIA [30] is a scheduler for Hadoop that meets deadlines using an analytical model of MapReduce to solve for the appropriate number of map and reduce slots. Jockey [8] is a similar system for more general dataparallel applications. Bazaar [15] translates these efforts to the cloud by transforming the typical resource-centric cloud API to a job-centric API whereby users request job deadlines rather than collections of VMs. In this model, the cloud provider applies the job profile to an analytical model to compute the cheapest way to meet the job's deadline.

Scale: In the public cloud, users are often presented with a choice of using a large number of slow, cheap VMs or a small number of fast, expensive VMs. The choice to scale out or scale up depends on the technology available. Michael et al. [21] compared a scale-up SMP server to a scale-out cluster of blades and found the scale-out configuration to 
be more cost effective. Half a decade later, Appuswamy et al. [2] revisited this question in the context of Hadoop and found the opposite to be true: that a single scale-up server is more cost-effective than a larger scale-out configuration.

While the relative costs of either approach change over time, scale-out configurations must be wary of excessive variance. Dean and Barroso [7] study tail latency in Web services at Google and demonstrate long-tailed latency distributions in production data centers. They call developers to build tail tolerance into their systems to avoid performance loss. Xu et al. [38] take a pragmatic approach and develop a system to screen for and remove outlier VMs in the long tail.

At the same time, Cockcroft [5] demonstrates how Netflix takes advantage of scale-up VMs on EC2 to reduce costs while substantially simplifying cluster configuration. Cockcroft relies on newer SSD-based VMs, indicating that available hardware drives the choice of whether to scale out or scale up. Of course, the software must also be capable of taking advantage of scale-up. Sevilla et al. [26] describe an optimization to MapReduce that alleviates I/O bottlenecks in scale-up configurations.

\section{Background}

We now present a brief overview of I/O resources in Amazon Web Services (AWS) and describe our application model.

\subsection{Amazon Elastic Compute Cloud}

Amazon Elastic Compute Cloud (EC2) is a cloud computing service that provides access to on-demand VMs, termed instances, at an hourly cost. There are many types of instances available, each with a particular mixture of virtual CPU cores (vCPU), memory, local storage, and network bandwidth. Table 1 lists a few examples.

VM instances are located in availability zones, which are placed across a variety of geographically distributed regions. VMs within the same region are engineered to provide lowlatency and high-bandwidth network access to each other. The cost of individual VMs varies by instance type, as well as over time, as new hardware is deployed within AWS. In this paper, we only consider "on-demand" pricing, representing the cost to reserve and keep instances during a given job. Finally, although the cloud offers the abstraction of unlimited computing and storage resources, in reality the number of resources in a given availability zone is limited. This complicates cluster provisioning because the most economical cluster for a given job might not be available when the user needs it. In our experience, launching even $100 \mathrm{VMs}$ of a specific type required two weeks of back and forth communication with engineers within Amazon. Even then, we were only permitted to allocate the virtual machines in a short window of a few hours.

\subsection{Virtualized I/O}

Recent advances in I/O-virtualization technology have made the cloud an attractive platform for data-intensive comput- ing. Here we discuss three types of virtualized I/O available in the cloud.

\subsubsection{Virtualized Storage}

In 2012, Amazon introduced the first EC2 VM with solidstate storage devices. Prior to this, all EC2 VMs ran either on disk or persistent network-attached storage. Over the next two years, more and more VMs with SSDs became available. By mid 2014, Amazon began highlighting its SSD offerings, relegating the disk-based VMs to the "Previous Generation" of VMs. Other cloud providers have followed suit in the race for newer and faster storage technologies. Google recently added a local SSD offering to its Compute Engine [11] cloud. Microsoft Azure's new G-series VMs include large amounts of local SSD storage [4].

Because offered bandwidth is so high and access times are so low, significant effort is required to support these devices in a virtualized environment at full speed. If the hypervisor spends too much time processing $\mathrm{I} / \mathrm{O}$ on shared devices, performance will suffer. Recent virtualization technologies, such as Single Root I/O Virtualization (SR-IOV), enable providers to expose a high-speed I/O device as many smaller, virtualized devices [28]. With SR-IOV, the hypervisor is out of the data path, enabling faster guest VM access to these devices.

\subsubsection{Virtualized Network}

Today, high-speed networks are common in public cloud platforms. EC2 has offered VMs connected to a $10 \mathrm{~Gb} / \mathrm{s}$ network as early as 2010, although these VMs were primarily targeted at scientific cluster computing. More recently, $10 \mathrm{~Gb} / \mathrm{s}$ networks have been rolled out to VM types targeting more general workloads. While achieving maximum network performance is difficult on dedicated hardware, virtualization adds another level of complexity that needs to be addressed for achieving efficiency. As in the case of storage, technologies such as SR-IOV can reduce virtualization overheads and make the most of the high speed network. In a shared environment, SR-IOV can be used to slice the $10 \mathrm{~Gb} / \mathrm{s}$ interface so each VM receives a portion of the bandwidth. In the case of a single guest VM, eliminating overhead makes $10 \mathrm{~Gb} / \mathrm{s}$ transfer speeds possible.

Amazon offers SR-IOV through a feature called enhanced networking. Though not all VMs support enhanced networking, a large portion of the newer VMs can access the feature. These include not only the VMs that support $10 \mathrm{~Gb} / \mathrm{s}$, but also their smaller counterparts, which are likely carved up from larger instance types using SR-IOV to efficiently share a single $10 \mathrm{~Gb} / \mathrm{s}$ NIC.

Enhanced networking also enables VMs to launch in a placement group. Placement groups instruct EC2 to provision VMs strategically in the network to increase bisection bandwidth. Given that oversubscription is common in large data center networks [13], placement groups play an important role in delivering high performance to the user. 


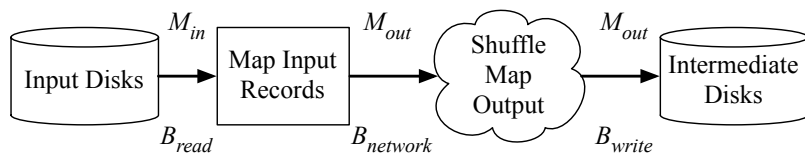

Figure 1: Themis phase 1: $\operatorname{map}()$ and shuffle.

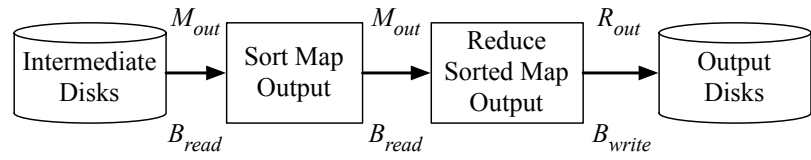

Figure 2: Themis phase 2: sort and reduce().

\subsubsection{Network-Attached Storage}

A third type of virtualized I/O, network-attached storage, is a common way to implement persistent storage in cloud environments. The local storage devices described in Section 3.2.1 are typically erased after a VM shuts down or migrates. To store persistent data, users are directed to separate storage services, such as Amazon Simple Storage Service (S3) or Amazon Elastic Block Store (EBS). These services are accessed remotely by a variety of interfaces. For example, S3 supports a RESTful API and can be accessed via HTTP, while EBS is exposed as a standard block device. In this work, we evaluate EBS because its interface is similar to a local storage device, supporting unmodified applications. To access EBS, users simply attach a volume to a running instance. Volumes can be created with near arbitrary size and IOPS requirements, backed either by disks or SSDs.

Achieving high performance on persistent, networkattached storage brings its own complexities. On the backend, the storage service must be provisioned with enough storage devices to suit users' needs and also have an efficient way of carving them up into volumes. Typically these storage services are also replicated, further increasing complexity. On the client's side, the black-box nature of the storage service complicates issuing optimal I/O patterns. Finally, congestion in the network or interference from co-located VMs can reduce performance in an unpredictable way.

\subsection{Application Models}

In this work, we focus on the performance of I/O-bound jobs and deploy Themis, our in-house MapReduce [22, 24]. Themis implements MapReduce as a two pass, pipelined algorithm. In its first map and shuffle pass (Figure 1), Themis reads input data from disk into small, in-memory buffers. It then applies the map () function to records in these buffers, and the resulting map output, or intermediate, data is divided into partitions. Unlike traditional MapReduce systems, which write intermediate data to local disk, Themis streams intermediate data buffers over the network to remote nodes before writing to partition files on the remote node's local disks. This implementation eschews traditional tasklevel fault tolerance in favor of improved I/O performance.
In the second sort and reduce pass (Figure 2), Themis reads entire intermediate partitions from local disk into memory. It then sorts these partitions and applies the reduce() function. Finally, the resulting records are written to output partition files on local disk. In the rare event that partitions do not fit in memory, a separate mechanism handles these overly large partitions.

We now model the performance of Themis under several assumptions about I/O efficiency and data durability.

\subsubsection{2-IO}

Because Themis eschews traditional task-level fault tolerance, it exhibits the 2-IO property [22], which states that each record is read from and written to storage devices exactly twice. In this paper, we consider data sorting as our motivating application. For external sorting, Themis achieves the theoretical minimum number of I/O operations [1]. This property not only makes Themis efficient, but it also yields a very simple computational model. When we restrict our focus to I/O-bound applications, the processing time of the map and shuffle phase can be modeled as:

$$
T_{1}=\max \left(\frac{M_{\text {in }}}{B_{\text {read }}}, \frac{M_{\text {out }}}{B_{\text {network }}}, \frac{M_{\text {out }}}{B_{\text {write }}}\right)
$$

where $M_{\text {in }}$ and $M_{\text {out }}$ represent the per-node map input and output data sizes, and $B_{\text {read }}, B_{\text {write }}$, and $B_{\text {network }}$ represent the per-node storage and network bandwidths. For clarity, we have labeled these variables in Figures 1 and 2. In the particular case of sorting, map input and output are the same, and if we ensure that storage read and write bandwidths are the same, we are left with:

$$
T_{1}=\max \left(\frac{D}{B_{\text {storage }}}, \frac{D}{B_{\text {network }}}\right)
$$

where $D$ is data size to be sorted per node. Next we compute the processing time of sort and reduce phase. Because this phase involves only local computation, storage is the only I/O bottleneck:

$$
T_{2}=\max \left(\frac{M_{\text {out }}}{B_{\text {read }}}, \frac{R_{\text {out }}}{B_{\text {write }}}\right)
$$

where $R_{\text {out }}$ is the reduce output data size. Again in the case of sort, this is equal to $D$, the per-node data size, so the processing time is:

$$
T_{2}=\frac{D}{B_{\text {storage }}}
$$

In practice, it may not be the case that read and write bandwidths are equal, in which case we have:

$$
B_{\text {storage }}=\min \left(B_{\text {read }}, B_{\text {write }}\right)
$$

Therefore the final processing time of the sort is:

$$
T=T_{1}+T_{2}=\max \left(\frac{D}{B_{\text {storage }}}, \frac{D}{B_{\text {network }}}\right)+\frac{D}{B_{\text {storage }}}
$$

Finally, we account for the VM's hourly cost $C_{\text {hourly }}$ to compute the total dollar cost of the sort:

$$
C=C_{\text {hourly }}\left[\max \left(\frac{D}{B_{\text {storage }}}, \frac{D}{B_{\text {network }}}\right)+\frac{D}{B_{\text {storage }}}\right]
$$




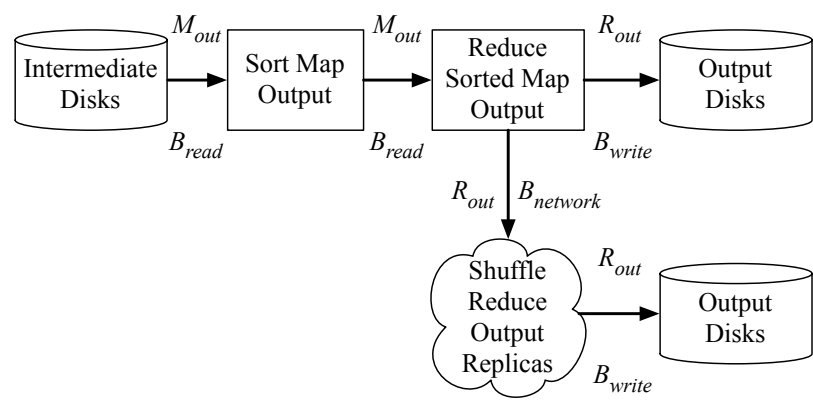

Figure 3: Sort and reduce() with Application-Level Replication.

\subsubsection{Application-Level Replication}

The 2-IO model represents the upper-bound of costefficiency and performance for I/O-bound jobs. In practice, storing only one copy of the data dramatically reduces durability. We now consider the case where the application makes a remote replica of each output file for improved durability.

We augment the sort and reduce phase with output replication as shown in Figure 3. In addition to writing output partitions to local output disks, the system creates a replica of each output file on a remote node's local output disks. This incurs an extra network transfer and disk write for each output partition file. This online replication affects the total processing time of the sort and reduce phase:

$$
T_{2}=\max \left(\frac{M_{\text {out }}}{B_{\text {read }}}, \frac{R_{\text {out }}}{B_{\text {network }}}, \frac{2 R_{\text {out }}}{B_{\text {write }}}\right)
$$

In the case of sort, this becomes:

$$
T_{2}=\max \left(\frac{D}{B_{\text {read }}}, \frac{D}{B_{\text {network }}}, \frac{2 D}{B_{\text {write }}}\right)
$$

Notice there is now an asymmetry in the storage bandwidth requirements between the map and shuffle phase (Equation 2) and the sort and reduce phase (Equation 9). This asymmetry will necessitate storage configuration changes, as we will see in Section 5.2.

\subsubsection{Infrastructure-Level Replication}

Implementing Application-Level Replication as described in Section 3.3.2 adds significant complexity and cost. Cloud providers typically offer infrastructural services to reduce the burden on application developers.

To illustrate the use of Infrastructure-Level Replication, we consider running Themis MapReduce on Amazon EC2 using the EBS storage service described in Section 3.2.3 for input and output data, and local disks for intermediate data only. The time for the map and shuffle phase becomes:

$$
T_{1}=\max \left(\frac{M_{\text {in }}}{B_{\text {readEBS }}}, \frac{M_{\text {out }}}{B_{\text {network }}}, \frac{M_{\text {out }}}{B_{\text {write }}}\right)
$$

Similarly, the time for sort and reduce is:

$$
T_{2}=\max \left(\frac{M_{\text {out }}}{B_{\text {read }}}, \frac{R_{\text {out }}}{B_{\text {writeEBS }}}\right)
$$

We consider the performance and cost implications of these three models in the sections that follow. Section 4 thoroughly explores the 2-IO model, while Section 5 describes a large-scale evaluation of all three models.

\subsubsection{Model Generality}

While our models cover several use cases, we acknowledge that they are specific to Themis MapReduce. We now discuss how these models might generalize to other platforms.

Themis MapReduce derives much of its I/O-efficiency from its pipelined implementation, which limits the amount of extraneous I/O relative to frameworks like Hadoop [39]. MapReduce Online [6] brings pipelining to Hadoop, although extra disk I/O is still required for fault tolerance. In general, our models could be updated to handle additional I/O operations performed by other frameworks.

In this work, we assume that $\mathrm{I} / \mathrm{O}$-intensive jobs are $\mathrm{I} / \mathrm{O}$ bound. While compute-bound jobs are outside the scope of this work, we posit that a new model could be constructed from the job's compute requirements. Such a model could include terms for both $\mathrm{I} / \mathrm{O}$ and compute time.

Our models assume zero scheduling overheads because Themis runs one jobs at a time and one process per node per phase of that job. In general, the models could be updated to account for framework scheduling overheads.

Finally, many I/O-intensive applications run frameworks that are not MapReduce. For example, computation over large graphs may benefit from specialized frameworks [18, 19]. These frameworks will require different computational models, but should include some similar terms for I/Ointensive jobs and will likely benefit from the techniques described in this work.

\section{Profiling AWS Storage and Networking}

We now turn our attention to choosing a cluster configuration on EC2 for I/O-bound applications. As we will show, it is not simply enough to know the VM specifications. The scaling behavior of each VM must be taken into account.

To this end, we design experiments to estimate the performance of I/O-bound jobs on EC2. First, we measure the per-VM bandwidth of local storage (Section 4.2). This approximates job performance when the network is not the bottleneck $\left(B_{\text {network }}=\infty\right.$ in our models). Next, we measure the network performance of each VM type (Section 4.3). Together, these metrics give a performance estimate that accounts for either bottleneck, but assumes that network performance scales perfectly. Then, we measure the actual scaling behavior of the network using larger cluster sizes to refine this performance estimate. Finally, we combine these results with published hourly costs to select the most costeffective VM for running a large-scale $100 \mathrm{~TB}$ sort job under the 2-IO model described in Section 3.3.1.

We run the analysis using two custom-built microbenchmark tools: (1) DiskBench, which measures the overall 
throughput of the storage subsystem within a single node, and (2) NetBench, which measures network performance by synthetically generating data without involving local storage $^{1}$. We describe these tools in the sections that follow.

\subsection{Measurement Limitations}

A common concern when conducting measurements of the public cloud is variance. Resource sharing between customers, either co-located on the same machine or utilizing the same network, increases variance and makes measurement more difficult. Getting a completely fair assessment of the performance of the cloud is complicated by diurnal workload patterns that necessitate measuring at different times of day. Jobs launched during business hours may cause weekends and weekdays to see differing levels of performance. Less-frequent, periodic jobs may even lead to changes based on week of the month or month of the year.

In addition to user-created variance, the underlying infrastructure is constantly changing, meaning that any measurement is just a snapshot of the cloud in its current state. For example, in the time between the experiments in this paper and its publication, Amazon has added 16 new instance types to EC2, all of which can affect the performance of the shared network. Variance can even exist between different data centers belonging to the same provider. Different data centers may contain I/O devices with different performance levels, as Schad et al. [25] have shown.

While we acknowledge such variance exists, we admit that our ability to quantify it is limited. Despite partial support from Amazon's educational grant program, the experiments in this paper totaled more than $\$ 50,000$ in AWS costs, and so we were not able to continue studying AWS in enough detail to account for these forms of variance.

Much of this cost comes from running large-scale benchmarks and evaluations to tease out the scaling properties of network. Someone with more insight into the networking properties of the cloud, e.g. the provider, might be able to factor this into the models described in Section 3.3, eliminating a large portion of the costs and freeing up resources for in-depth variability analysis.

Finally, it is not always possible to allocate a large number of on-demand VMs. The large-scale evaluations in Section 5 were only possible after weeks of back-and-forth communication with AWS engineers. When we were finally able to allocate the VMs, we were instructed to decommission them after only a few hours, proving further measurement impossible. For these reasons, a comprehensive study of variance in the cloud is not presented in this work.

\subsection{Local Storage Microbenchmarks}

We begin our measurement study by profiling the local storage available on each EC2 VM type with DiskBench. Because local storage devices are often faster than network-

\footnotetext{
$\overline{{ }^{1} \text { Available at http://themis.sysnet.ucsd.edu }}$
}

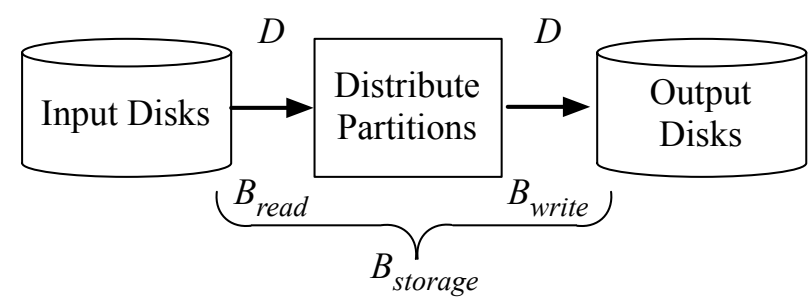

Figure 4: The DiskBench storage microbenchmark runs locally on a single node without involving the network.

attached storage, these measurements are typically an upperbound on storage performance. We revisit the choice of local versus remote storage in Section 4.4.

\subsubsection{The DiskBench Microbenchmark}

DiskBench, shown in Figure 4, is a pipelined application that reuses many of the components of Themis MapReduce (Section 3.3). DiskBench isolates the storage subsystem from map and shuffle phase by reading records without applying the $\operatorname{map}($ ) function. Records are randomly assigned to partitions on the same node, and are written back to local disk without involving a network shuffle.

We configure DiskBench to use one half of the local disks for reading and the other half for writing for VMs with more than one disk. This configuration is typically ideal for local storage devices, and is in fact the configuration used in our earlier experience with high speed sorting [24]. As a result, the bandwidths reported by DiskBench measure a read/write workload, and in many cases are approximately half of the bandwidth available in read-only or write-only workloads.

\subsubsection{Experimental Design}

We instantiate two to three VMs of each type offered by AWS in the us-east-1a availability zone. We run DiskBench three times on each of these clusters and compute the average per-node storage bandwidth, $B_{\text {storage }}$, measured in megabytes per second (MB/s). We run DiskBench on multiple instances to account for natural variance in performance between VMs.

\subsubsection{Analysis}

The results of DiskBench are shown in Figure 5. We report the mean read/write storage bandwidth, as well as the offered per-VM storage capacity. We group VMs into regions based on cluster size needed to sort 100 TB of input data; the rightmost region represents instance types needing fewer than 100 VMs. The middle region represents types needing between 100 and 1,000 VMs. Finally, the leftmost region represents types needing more than 1,000 VMs. We highlight these regions because provisioning large clusters is not always possible. For example, we found that even with the help of Amazon's engineers, we were only able to allocate at most 186 instances of $i 2.8 x$ large in a single availability 


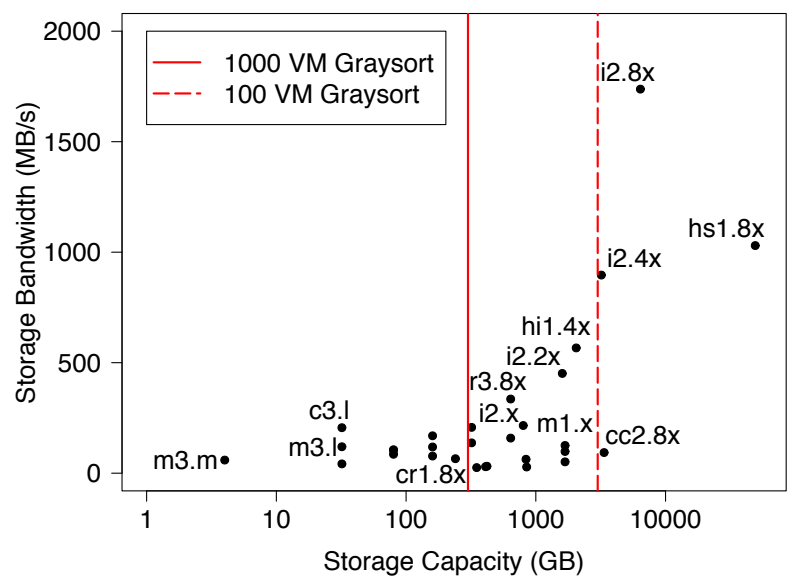

Figure 5: Storage performance of EC2 VMs reported by DiskBench. Vertical lines cluster VM types into those requiring more than 100 or 1,000 instances to sort 100 TB.

zone. Furthermore, as we will show, network performance can degrade significantly with larger clusters.

In Figure 5 we have labeled some of the more interesting instance types. Many of these are on the upper right-hand side of the figure and represent a candidate set of instance types which deliver both high storage performance and host enough local storage to meet the capacity requirements of a 100 TB sort with a small cluster. The highest performing instance type in the sub- $100 \mathrm{VM}$ region is i2.8xlarge, which contains eight 800 GB SSDs and offers $1.7 \mathrm{~GB} / \mathrm{s}$ of simultaneous read/write bandwidth as measured by DiskBench. The i2.4xlarge instance type has half the number of SSDs, with half as much storage bandwidth as a result. Another interesting instance type is $\mathrm{hs} 1.8 \mathrm{xlarge}$, which provides the highest density of storage using HDDs instead of SSDs. The hs1.8xlarge instance type includes 24 local HDDs and supports $1.06 \mathrm{~GB} / \mathrm{s}$ of read/write bandwidth. Because of its high storage density, only seven instances are needed to meet the capacity needs of a $100 \mathrm{~TB}$ sort operation.

Estimating the dollar cost of sorting: We next use the results of DiskBench and the listed AWS hourly cost to predict the total dollar cost of running a 100 TB 2-IO sort using Themis. Here, we consider only local storage performance $\left(B_{\text {network }}=\infty\right)$, and apply the results from Figure 5 to Equation 7 to estimate the total cost of sorting 100 TB.

Table 2 shows a subset of these results ranked by total sorting cost. To highlight capacity limitations, we compute the number of VMs required to hold $300 \mathrm{~TB}$, which covers the input, intermediate, and output data for a 100 TB sort.

From Table 2, we see that c3. large is the most economical, with a per-sort cost of $\$ 28$. However, each VM only has $32 \mathrm{~GB}$ of storage, so 9,375 instances are required to hold the necessary $300 \mathrm{~TB}$ of data. Next are the m3. large and $\mathrm{m} 3$.medium instance types, with a sort cost of approximately $\$ 65$. Again, scaling to meet capacity requirements is a significant challenge. In fact, it is not until the $\mathrm{m} 1$ in-

\begin{tabular}{|c|c|c|c|}
\hline \multirow[t]{2}{*}{ Instance } & \multirow{2}{*}{$\begin{array}{l}\text { Min. nodes } \\
\text { required for } \\
\text { 100TB sort }\end{array}$} & \multicolumn{2}{|c|}{ Cost } \\
\hline & & $\begin{array}{r}\text { Sort } \\
(\$)\end{array}$ & $\begin{array}{r}\text { Hourly } \\
(\$ / h r)\end{array}$ \\
\hline c3.lar & 9,375 & 28 & 0.105 \\
\hline m3. & 9,375 & 65 & 0.14 \\
\hline m3.m & 75,000 & 66 & 0.07 \\
\hline m1.xlar & 179 & 155 & 0.35 \\
\hline $\mathrm{i} 2.4 \mathrm{xl}$ & 94 & 211 & 3.41 \\
\hline i2.8xlarge & 47 & 218 & 6.82 \\
\hline hs1.8xlarge & 7 & 248 & 4.60 \\
\hline cr1.8xlarge & 1,250 & 2,966 & 3.50 \\
\hline
\end{tabular}

Table 2: Estimated dollar cost of sorting $100 \mathrm{~TB}$ on a subset of EC2 instance types based solely on local storage performance.

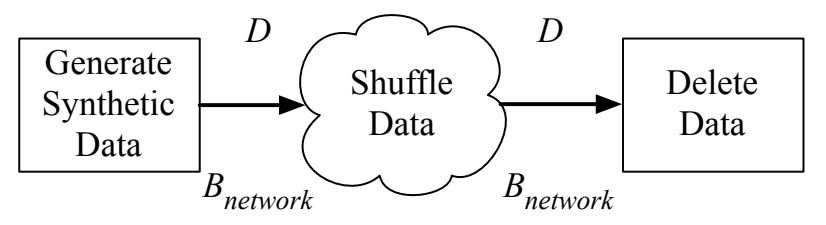

Figure 6: The NetBench network microbenchmark measures network scalability and performance using synthetic input data.

stance types that clusters of $O(100)$ nodes will suffice. The first instance types with $O(10)$ node cluster sizes are the i2 types, which are built with arrays of SSDs. A $100 \mathrm{~TB}$ sort can be completed with just 47 i2.8xlarge instances at a cost of $\$ 218$. For reference, the most expensive instance type is cr $1.8 x$ large, a memory-optimized 32-core instance type with two 120 GB SSDs, on which a 100 TB sort would cost $\$ 2,966$, a factor of over 100x more expensive than the cheapest instance type. It is worth noting that two instance types might have hourly costs that are an order of magnitude apart, but the total cost to the user may be very similar, e.g., $\mathrm{m} 1 . \mathrm{xlarge}$ and i2.4xlarge.

Summary: Measuring VM storage bandwidth provides great insight into the total cost of a large-scale data-intensive application. Many high-performance VM configurations can deliver reasonable costs using a small number of nodes.

\subsection{Network Microbenchmarks}

Next, we measure the performance and scalability of the AWS networking infrastructure. We focus on the subset of instance types that have relatively high performance and high storage capacity as measured in Section 4.2.

\subsubsection{The NetBench Microbenchmark}

Similar to DiskBench, NetBench (Figure 6) is a pipelineoriented application derived from Themis. Following the analogy, NetBench aims to isolate the network subsystem from the map and shuffle phase. Synthetic data records are generated in-memory and shuffled over the network to remote nodes, which simply delete the data records. NetBench operates entirely in memory and does not touch local disk. 


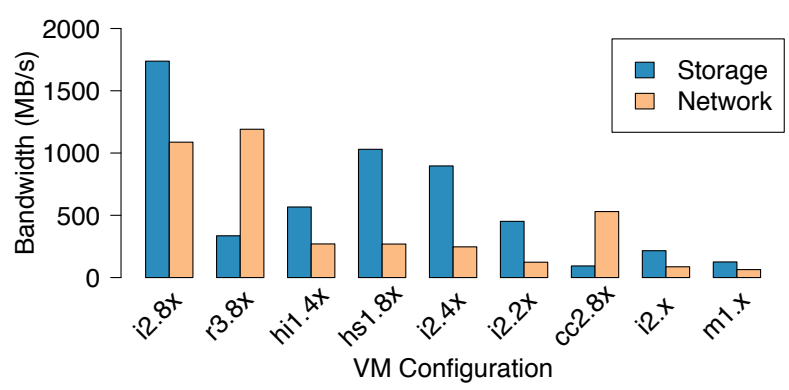

Figure 7: Comparison between storage and network performance.

\subsubsection{Experimental Design}

We perform two experiments to measure the AWS networking infrastructure. The first experiment determines the baseline network bandwidth of each instance type. For each VM type, we allocate a cluster of two nodes in the us-east-1a availability zone. On each of these clusters, we run NetBench three times. From these three data points, we compute the average observed network bandwidth, $B_{\text {network }}$, which we report in the unconventional unit of megabytes per second $(\mathrm{MB} / \mathrm{s})$ for easy comparison with the results of DiskBench. This measurement represents the ideal scaling behavior of the network. When available, we enable the enhanced networking feature and allocate nodes in a single placement group, and we use two parallel TCP connections between nodes to maximize the bandwidth of the high speed VMs.

Next, we quantify the network scaling behavior in a candidate set of VM types. For each type, we construct increasingly larger clusters by allocating two VMs to get a cluster of size two, allocating two more to get a cluster of size four, allocating four more to get a cluster of size eight, and so on. On each cluster, we run NetBench once and measure the all-to-all network bandwidth observed by the slowest VM.

The largest measured cluster varies by instance type. In many cases, limits imposed by AWS prevented larger study. For some of the more expensive VMs, we cap the maximum cluster size due to limited funds. We do not use placement groups in this experiment because doing so alters the natural scaling behavior of network and limits cluster sizes. Placement groups also work best when all VMs launch at the same time. This launch pattern is neither representative of elastically scaling applications, nor is it applicable to our experiment setup. Additionally, we use a single TCP connection between VMs because using multiple TCP connections reduces performance at larger cluster sizes, and we are ultimately interested in the performance at scale.

\subsubsection{Analysis}

We show the baseline network performance of a select subset of VMs in Figure 7. For comparison, we also show the storage performance measured in Section 4.2. For many VMs, the storage and network bandwidths are mismatched. Equation 2 (Section 3.3) suggests that we want equal amounts of storage and network bandwidth for the map and shuffle

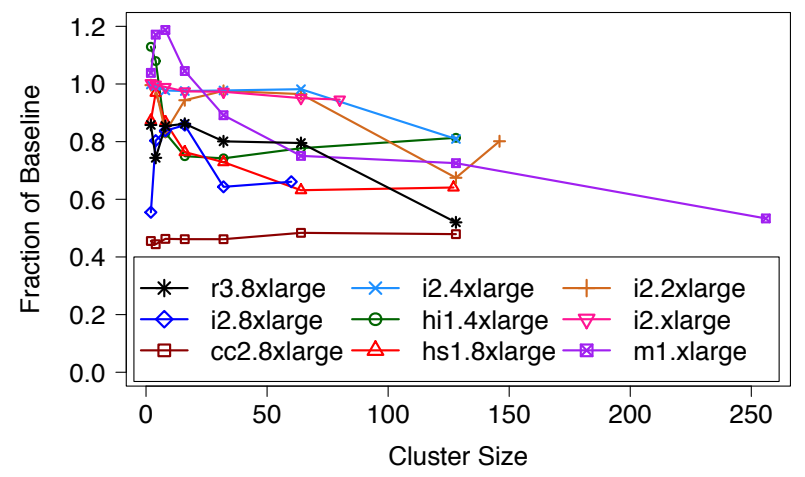

Figure 8: Network performance scalability displayed as a fraction of the baseline network performance given in Figure 7.

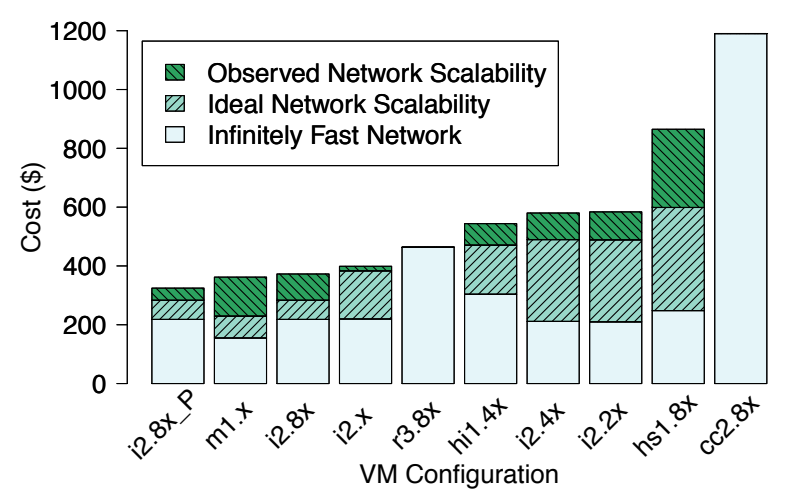

Figure 9: Estimated cost of sorting 100 TB on a subset of EC2 VM types, under various network performance assumptions.

phase of sort, but this is often not achieved. For example, the network bandwidth of i2.8xlarge is only $63 \%$ of its measured storage bandwidth. This mismatch reduces the end-toend performance of an application that must use both storage and network $\mathrm{I} / \mathrm{O}$, resulting in underutilized resources.

Figure 8 illustrates the scaling behavior of the network, displayed as a fraction of the baseline bandwidth. This comparison is not perfect because the experiments were run on different sets of VMs on different days during a two week period and at different times of day. This perhaps explains how $\mathrm{m} 1 . \mathrm{xlarge}$ and $\mathrm{hi1} .4 \mathrm{xlarge}$ reach speeds that are $20 \%$ faster than the baseline at small cluster sizes.

However, the main takeaway is that performance degrades significantly as more nodes are added to the cluster. In eight of the nine VM types measured, performance drops below $80 \%$ of baseline during the experiment. One instance type, cc2.8xlarge, shows consistently poor performance. We speculate this type resides in a highly congested portion of the network and can only achieve high performance when placement groups are enabled.

The dollar cost of sorting revisited: Finally, we use the results of DiskBench and NetBench to predict the total monetary cost of running a $100 \mathrm{~TB} 2-\mathrm{IO}$ sort operation on each of the VM instance types. We apply our measurements to Equation 7 (Section 3.3) to determine the total dollar cost. 
Predicted costs are shown in Figure 9. For the selected VMs, we show (1) the total cost assuming that the network is not the bottleneck, (2) the cost assuming that network bandwidth scales in an ideal manner, and (3) the cost based on the observed scale-out networking performance. The results show that the lowest-cost VM for sort is $\mathrm{m} 1$. $\mathrm{xlarge}$, at $\$ 362$ per sort followed closely by i2.8xlarge and i2.xlarge. Interestingly, while the ideal network scalability cost of i2.8xlarge is greater than $\mathrm{m} 1 . \mathrm{xlarge}$, $\mathrm{i2.8xlarge} \mathrm{has}$ better actual network scaling properties, resulting in very similar overall dollar costs. However, the i2.8xlarge instance type supports placement groups, which if employed actually result in a lower overall cost than $\mathrm{m} 1 . \mathrm{xlarge}$. We represent this configuration as $i 2.8 x_{P} P$, with an estimated cost of $\$ 325$, which is $\$ 37$ cheaper than $\mathrm{m} 1$. $\mathrm{xlarge}$.

Summary: Networking performance, particularly at scale, must be accounted for when estimating cost. Poor scaling performance can significantly drive up costs. Better network isolation, e.g. placement groups, can substantially reduce costs. In the case of sort, network isolation results in a savings of $\$ 37$, or about $10 \%$.

\subsection{Persistent Storage Microbenchmarks}

We now turn our attention to network-attached storage. While local storage typically has better performance, many cloud deployments will want input and output data to persist across VM resets and migrations. We now consider the performance properties of Elastic Block Store (EBS), a persistent network-attached storage service offered by AWS.

\subsubsection{Experimental Design}

To measure the performance of EBS, we allocate three i2. $4 x$ large instances in us-east-1a with the enhanced networking and EBS-optimization features enabled. At the time of the experiment, i2.4xlarge was one of the few VM types supporting a maximum EBS throughput of $250 \mathrm{MB} / \mathrm{s}$. As of this writing, Amazon offers three new instance types with speeds of up to $500 \mathrm{MB} / \mathrm{s}$. EBS offers three types of storage volumes: magnetic disk, general purpose SSDs, and IOPS-provisioned SSDs. For each type, we create and attach eight 215 GB EBS volumes to each of the three i2. 4 xlarge instances. We then run DiskBench, and vary the number of EBS volumes used.

We configure DiskBench to run in read-only and writeonly modes, but not in the read/write mode described in Section 4.2.1. This more closely resembles an actual EBSbacked application, which will read input data from persistent storage, process it using local per-VM storage, and then write output data back to persistent storage. This usage pattern directly corresponds to the Infrastructure-Level Replication model described in Section 3.3.3.

We run each combination of EBS volume type, number of EBS volumes, and DiskBench mode three times on each of the three nodes to get an average bandwidth measurement.

\subsubsection{Analysis}

Figures 10a and 10b show the read-only and write-only results. There are four key points. First, a single EBS volume cannot saturate the link between the VM and EBS. Bandwidth increases as more volumes are added up to the $250 \mathrm{MB} / \mathrm{s}$ limit. Second, near-maximal read performance can be achieved using as few as three volumes of any type. Third, near-maximal write performance can be achieved using three SSD-based volumes. Finally, magnetic disk cannot achieve maximal write bandwidth with even eight volumes.

We conclude that EBS-optimized VMs can achieve maximal speeds on SSD-based volumes. However, EBS volumes are far slower than local, per-VM storage. For example, Figure 5 shows i2.4xlarge is capable of nearly $900 \mathrm{MB} / \mathrm{s}$ of $\mathrm{read} / \mathrm{write}$ bandwidth to its local SSDs. As such, EBS bandwidth is likely to be a bottleneck for Infrastructure-Level Replication (Equations 10 and 11) and will shift the cost analysis quite a bit from that derived in Section 4.3.3.

Summary: Persistent storage systems built from SSDs can deliver reasonable levels of storage performance. However, local, per-VM storage provides far higher levels of performance, so persistent storage will likely be a bottleneck.

\subsection{Key Takeaways}

For clarity, we highlight four key takeaways:

1. DiskBench can be used to cheaply assess the storage performance of a wide variety of VMs at a small-scale to estimate job cost. Smaller VM types deliver low levels of cost, but require many thousands of VMs. Larger VMs deliver reasonable levels of cost with orders of magnitude fewer VMs. VMs deemed too expensive can be removed from further consideration.

2. NetBench can be used to refine this cost analysis, taking into account network performance at both small and large scales. Many VMs exhibit poor network scaling properties, leading to estimated costs far larger than back-ofthe-envelop calculations based on VM specifications.

3. The use of i2.8xlarge with placement groups results in the best performance and also delivers the lowest estimated sorting cost using a small number of VMs. Other VMs, such as hs1.8xlarge, look great initially, but perform poorly in practice, resulting in a $3 \mathrm{x}$ cost-increase.

4. SSD-backed EBS volumes can deliver full performance for applications that need enhanced data durability. However, EBS is far slower than local storage on some VMs, and will likely be a bottleneck.

\section{Evaluation}

Thus far we have measured the I/O performance and scalability of several cloud offerings in AWS in the context of the 2-IO model described in Section 3.3.1. We now present a large-scale evaluation of 2-IO, as well as the other models presented in Section 3. We consider the problem of sort- 


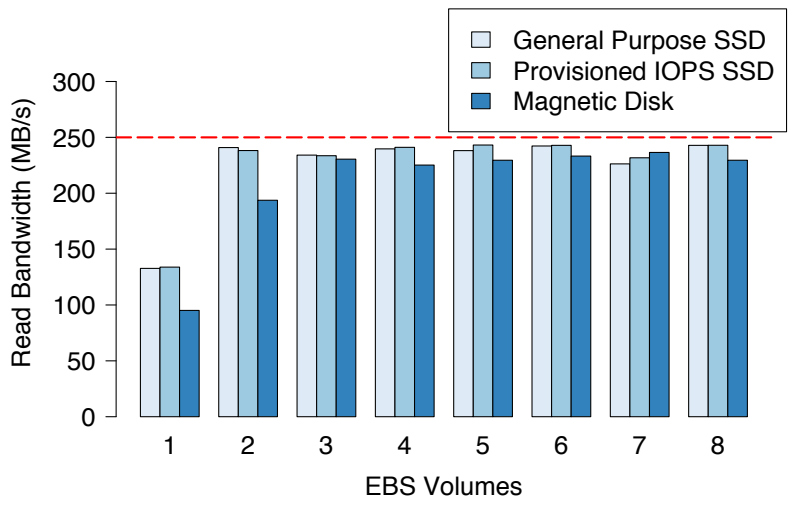

(a) EBS read performance.

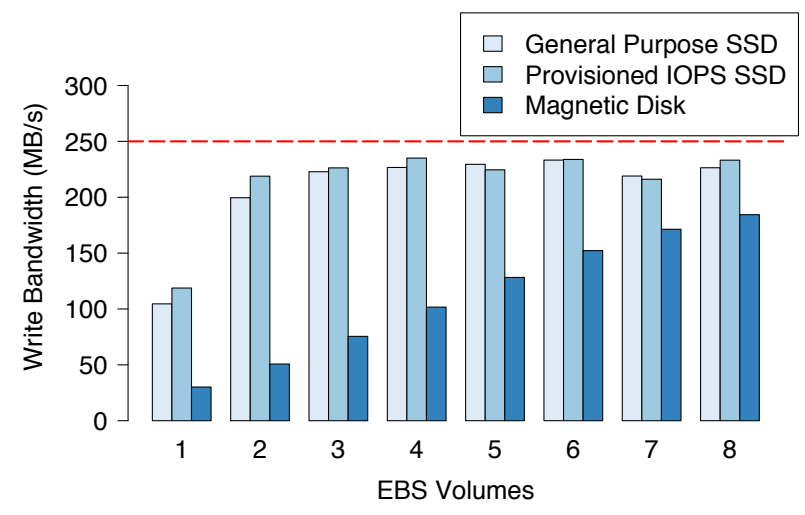

(b) EBS write performance.

Figure 10: EBS performance observed by i2.4xlarge. The maximum advertised performance is shown with a dashed line.

ing $100 \mathrm{~TB}$ and measure the performance and cost in each case. Each evaluation corresponds to one of a larger number of established large-scale sorting benchmarks [27], and thus represents a realistic problem that one might want to solve using the public cloud.

\subsection{2 -IO}

We evaluate the performance and cost of 2-IO by sorting a 100 TB data set that consists of one trillion 100-byte keyvalue pairs. Each pair consists of a 10-byte key and a 90byte value. Keys are uniformly distributed across the space of $256^{10}$ possible keys.

Experiment Setup: We allocate 178 on-demand instances of i2.8xlarge in a single placement group in the us-east-1a availability zone. We use local, per-VM SSDs for input, intermediate, and output data sets.

Before running the sort application, we run the DiskBench and NetBench microbenchmarks on the cluster to get a baseline performance measurement, and also to decommission VMs with faulty or slow hardware. DiskBench reports read/write storage bandwidth at $1515 \mathrm{MB} / \mathrm{s}$ for the slowest VM, which is $87 \%$ of the bandwidth measured in Section 4.2. NetBench yields a network bandwidth of $879 \mathrm{MB} / \mathrm{s}$ which is $81 \%$ of the baseline measured in Section 4.3. We note that this experiment was conducted on a different day than those in Section 4, and therefore may have somewhat different performance characteristics.

As in Section 4.2.1 we configure Themis to use four of the eight local SSDs for input and output files, and the remaining four SSDs for intermediate files.

Results: The 100 TB 2-IO sort completes in 888 seconds and requires $\$ 299.45$. To better understand the bottlenecks and limitations of this particular job, we collect system-level performance metrics using sar, iostat, and vnStat [29, 31]. Using these measurements, we find that during the approximately 500 seconds required to complete the map and shuffle phase, Themis is network-bound. Figure 11a shows the network utilization for three randomly chosen servers as a function of time. The $10 \mathrm{~Gb} / \mathrm{s}$ network is

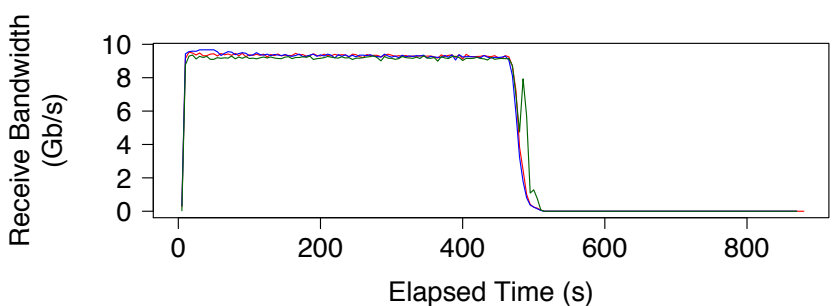

(a) Network receive throughput.

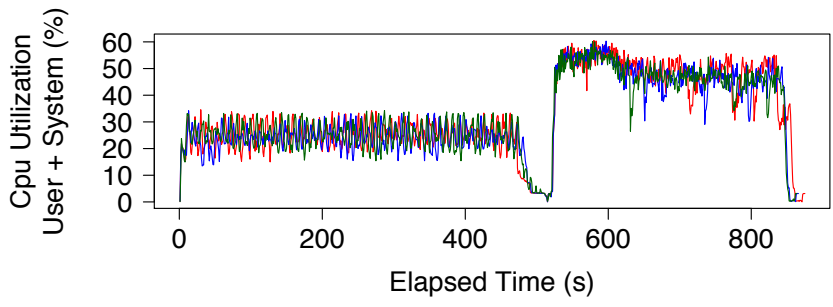

(b) CPU utilization.

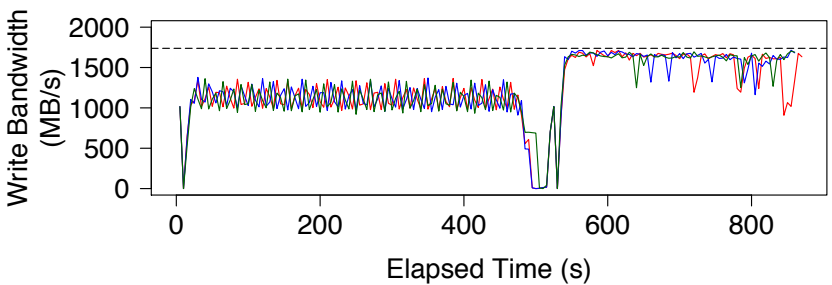

(c) Disk write bandwidth. The maximum hardware speed is denoted by a dashed line.

Figure 11: Resource usage of three VMs running the 100 TB 2-IO sort. The bottleneck shifts from network to storage at $t \approx 500 \mathrm{~s}$.

almost fully utilized, and as a result, the CPU and SSDs are only lightly utilized, as shown in Figures $11 \mathrm{~b}$ and $11 \mathrm{c}$.

The sort and reduce phase, which begins immediately after the map and shuffle phase completes, is I/O-bound by the local SSDs. Because no network transfer occurs in this phase, Themis can fully utilize the available storage bandwidth, and Figure 11c shows that the disk write bandwidth approaches the limitations of the underlying hardware. Multiple sorting threads allow CPU usage to increase consid- 


\begin{tabular}{l|r|r|r|r}
$\begin{array}{l}\text { System } \\
\text { Name }\end{array}$ & $\begin{array}{r}\text { Cluster } \\
\text { Size }\end{array}$ & $\begin{array}{r}\text { Sort } \\
\text { Speed } \\
(\mathrm{TB} / \mathrm{min})\end{array}$ & $\begin{array}{r}\text { Per-node } \\
\text { Speed } \\
(\mathrm{MB} / \mathrm{s})\end{array}$ & $\begin{array}{r}\text { Total } \\
\text { Cost } \\
(\$)\end{array}$ \\
\hline Themis & 178 & 6.76 & 633 & 299.45 \\
Hadoop & 2,100 & 1.42 & 11 & $?$ \\
Baidu & 982 & 8.38 & 142 & $?$
\end{tabular}

Table 3: Our 100 TB Indy GraySort entry. Past and current record holders are shown for comparison.

erably. However the overall system does not become CPUlimited, as illustrated in Figure 11b.

Because the sort job is I/O-limited, the final cost (\$299.45) closely resembles the estimated cost given Section 4.3 for i2.8xlarge with placement groups $(\$ 325)$. We conclude that the methodology in Section 4 can predict the cost of I/O-bound jobs with reasonable accuracy.

Sort Benchmark: While the analysis thus far has been focused on cost-efficiency, raw performance is also a highlydesired feature. Our 100 TB 2-IO sort conforms to the guidelines of the Indy GraySort 100 TB sort benchmark [27], and achieves an overall throughput of $6.76 \mathrm{~TB} / \mathrm{min}$. Our sort is nearly five times faster than the prior year's Indy GraySort record [12] (see Table 3), while still costing less than $\$ 300$.

We attribute this result to both the methodology in this paper, and also to our Themis MapReduce framework. It is important, however, to note that it is not simply our codebase that yields high performance. In fact, our Indy GraySort speed was surpassed by Baidu [16] by more than $20 \%$ using a system derived from TritonSort [23, 24], which also exhibits 2-IO. Thus the 2-IO model of computation has powerful implications for performance as well as cost-efficiency.

\subsection{Application-Level Replication}

Next we evaluate Application-Level Replication on the same 100 TB data set described in Section 5.1. We run a variant of Themis that supports output replication as illustrated in Figure 3. This particular configuration conforms to the Daytona GraySort benchmark specification [27].

Experiment Setup: This time we allocate 186 ondemand instances of i2.8xlarge. As before, we launch all instances in a single placement group. However, due to insufficient capacity in us-east-1a, we use the us-east-1d availability zone.

As alluded to in Section 3.3.2, the storage asymmetry in Application-Level Replication necessitates a slight change in the configuration of Themis. Here we use five of the eight SSDs for input and output files and the remaining three for intermediate files. This configuration more evenly balances the storage and network requirements of the MapReduce job.

Results: The 100 TB Application-Level Replication takes 1,378 seconds and results in a total cost of $\$ 485.56$. While a comparison between this result and the 2-IO result in Section 5.1 is not completely fair due to different sets of resources used in different availability zones on different

\begin{tabular}{l|r|r|r|r}
$\begin{array}{l}\text { System } \\
\text { Name }\end{array}$ & $\begin{array}{r}\text { Cluster } \\
\text { Size }\end{array}$ & $\begin{array}{r}\text { Sort } \\
\text { Speed } \\
(\mathrm{TB} / \mathrm{min})\end{array}$ & $\begin{array}{r}\text { Per-node } \\
\text { Speed } \\
(\mathrm{MB} / \mathrm{s})\end{array}$ & $\begin{array}{r}\text { Total } \\
\text { Cost } \\
(\$)\end{array}$ \\
\hline Themis & 186 & 4.35 & 390 & 485.56 \\
Spark & 207 & 4.27 & 344 & 551.36 \\
Hadoop & 2,100 & 1.42 & 11 & $?$
\end{tabular}

Table 4: Our 100 TB Daytona GraySort record.

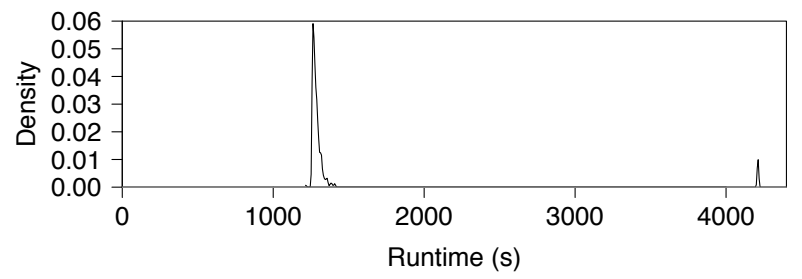

Figure 12: Bimodal elapsed times of reading 100 TB from EBS as seen by a cluster of 326 c $3.4 \times$ large VMs.

dates, it is nevertheless interesting to note that the improved data durability increases the cost of the sort from $\$ 299.45$ measured in Section 5.1 by $\$ 186.11$, or $62 \%$.

Sort Benchmark:The performance of our ApplicationLevel Replication surpassed the prior year's record-holder by more than $3 \mathrm{x}$, as seen in Table 4, setting the 100 TB Daytona GraySort record. Apache Spark, run by Databricks, submitted a benchmark result [35-37] that was slightly slower than ours, although our results are close enough to be considered a tie. However, our system is slightly more resourceefficient, resulting in a cost savings of $\$ 66$, or about $12 \%$.

It is interesting to note that Apache Spark also ran i2. $8 \times$ large, given that $\mathrm{EC} 2$ is not a requirement in this benchmark. At first glance, i2.8xlarge may look like an obvious choice for an I/O-intensive job like sorting. However, the results in this work show that performance at scale can differ substantially from initial estimates. For example, hs $1.8 \times 1$ arge also appears to be an obvious choice, despite its poor performance at scale, as shown in Section 4.

\subsection{Infrastructure-Level Replication}

Finally, we evaluate Infrastructure-Level Replication on the same $100 \mathrm{~TB}$ data set by running the 2-IO implementation of Themis using EBS volumes for input and output. This configuration meets the specifications for the Indy and Daytona CloudSort benchmarks [27], which measure the dollar cost of sorting on the public cloud.

Preliminary Results: EBS changes the cost analysis in Section 4, and our measurements suggest the cheapest VM type is $c 3.4 x$ large. We allocate $326 c 3.4 x l$ arge VMs in a single placement group in the us-east-1a availability zone and attach to each four 161 GB general purpose SSD EBS volumes. Unfortunately, this configuration experiences significant variance in read performance. Figure 12 shows a probability density function of runtimes across the 1,304 EBS volumes experienced when reading 100 TB from EBS. 


\begin{tabular}{l|r|r|r|r}
$\begin{array}{l}\text { System } \\
\text { Name }\end{array}$ & $\begin{array}{r}\text { Cluster } \\
\text { Size }\end{array}$ & $\begin{array}{r}\text { Sort } \\
\text { Time } \\
(\mathrm{s})\end{array}$ & $\begin{array}{r}\text { Per-node } \\
\text { Speed } \\
(\mathrm{MB} / \mathrm{s})\end{array}$ & $\begin{array}{r}\text { Total } \\
\text { Cost } \\
(\$)\end{array}$ \\
\hline Themis & 330 & 2981 & 102 & 450.84
\end{tabular}

Table 5: Our 100 TB Indy and Daytona CloudSort record.

Approximately $95 \%$ of the nodes complete in under 1,400 seconds, but the remaining nodes take three times longer. This long-tailed distribution makes c3.4xlarge an ineffective choice for Infrastructure-Level Replication at scale.

Experiment Setup: The next best option after c3.4xlarge is $\mathrm{r} 3.4 \mathrm{xlarge}$, which is $60 \%$ more expensive and offers approximately the same projected performance. We allocate $330 \mathrm{r} 3.4 \mathrm{xlarge}$ instances in a single placement group in the us-east-1c availability zone. We use a different zone because, as stated earlier in this work, it is often not possible to allocate a large number of instances in a particular zone. To each instance we attach eight $145 \mathrm{~GB}^{2}$ general purpose EBS volumes. We use EBS for input and output data and local SSD for intermediate data, as suggested in Sections 3.3.3 and 4.4.

Results: We run the sort three times and report an average completion time of 2,981 seconds and an average cost of $\$ 450.84$ (Table 5). This completion time, which includes two full rounds of I/O to EBS, is far less than the 4000 seconds required to run a single round of $\mathrm{I} / \mathrm{O}$ on a comparably sized cluster of $c 3.4 \times 1$ arge (Figure 12). We conclude that $r 3.4 x$ large does not experience the same longtailed behavior. The black-box nature of EBS prohibits further analysis, but one hypothesis is that the network connecting c $3.4 \mathrm{xlarge}$ to EBS is more congested, and thus more variable, than that of $r 3.4 x$ large. It may also be possible that the us-east-1c availability zone itself experiences better EBS performance at scale.

We note that the per-VM throughput is nearly half of the maximum $250 \mathrm{MB} / \mathrm{s}$ throughput to EBS. This indicates that each phase of the sort is running at near-optimal EBS speeds. In fact, Section 4.4 pins the ideal read and write bandwidths at 243 and $226 \mathrm{MB} / \mathrm{s}$, respectively. This suggests an ideal end-to-end throughput of $117 \mathrm{MB} / \mathrm{s}$, so our sort speed is $87 \%$ of optimal.

Sort Benchmark: These results set the world records for both Indy and Daytona CloudSort. Although far slower than Daytona GraySort in terms of performance, our CloudSort records actually sort $100 \mathrm{~TB}$ about $\$ 35$, or about $8 \%$, cheaper with even stronger durability requirements.

\section{Extending to Other Clouds}

While an analysis of other cloud providers is outside the scope of this work, it is desirable for our results to extend to other clouds. We now briefly discuss Google Compute Engine [11] and consider how our work might generalize.

\footnotetext{
${ }^{2}$ Actually $135 \mathrm{GiB}$. The EBS API uses GiB $\left(2^{30}\right)$ rather than GB.
}

\begin{tabular}{l|r|r|r} 
Type & vCPU & RAM (GB) & Cost $\mathbf{( \$ / h r )}$ \\
\hline n1-standard-1 & 1 & 3.75 & 0.05 \\
n1-standard-8 & 8 & 30 & 0.40 \\
n1-highcpu-32 & 32 & 28.8 & 1.216 \\
n1-standard-32 & 32 & 120 & 1.60 \\
n1-highmem-32 & 32 & 208 & 2.016
\end{tabular}

Table 6: Five example Compute Engine VMs.

Compute Engine offers VMs much like EC2. As of this writing, there are $18 \mathrm{VM}$ types divided into four categories, and we list a subset in Table 6 .

VMs do not, by default, have any local storage. Persistent disk, a service akin to EBS, is the default storage model. Users can optionally add a limited number of local SSDs to a VM for a price. It is not possible to add local HDDs.

Our limited experience shows that Themis, DiskBench, and NetBench, can achieve high levels of performance at a small-scale on a variety of VM types. Compute Engine currently lacks an explicit network placement mechanism. This may affect the network performance observed by a large number of VMs, although further analysis is required to make this claim.

\section{Conclusions}

High-speed flash storage and $10 \mathrm{~Gb} / \mathrm{s}$ virtualized networks supporting SR-IOV have enabled high performance dataintensive computing on public cloud platforms, and yet achieving efficiency remains challenging for these workloads. We present a systematic methodology for measuring the I/O capabilities of high-performance VMs, and extensively measure these features within EC2 using our custom microbenchmark tools, DiskBench and NetBench. We find that expected costs rise dramatically due to poor network scaling, altering the optimal choice of VM configurations. By provisioning based on performance measurements at scale, we demonstrate highly efficient sorting on EC2 and set three new world records at very low cost.

\section{Acknowledgments}

We wish to thank Geoff Voelker, Alexander Rasmussen, Rishi Kapoor, and our shepherd, Tyson Condie, for their valuable feedback while writing this paper. We would also like to thank Praveen Gujar, Chad Schmutzer, Ann Merrihew, Amy Hogenhurt, Jonathan Fritz, and Rahul Pathak from Amazon for their help in provisioning EC2 resources and obtaining EC2 credits. Finally, we wish to thank the sort benchmark organizers, Mehul Shah, Chris Nyberg, and Naga Govindaraju. This project was sponsored in part by the Amazon Educational Grant program, by the UCSD Center for Networked Systems, and by grants from the National Science Foundation (CNS-\#116079 and CNS-0964395). This project was supported by a donation from FusionIO. 


\section{References}

[1] A. Aggarwal and J. Vitter. The input/output complexity of sorting and related problems. CACM, 31(9), Sept. 1988.

[2] R. Appuswamy, C. Gkantsidis, D. Narayanan, O. Hodson, and A. Rowstron. Scale-up vs scale-out for Hadoop: Time to rethink? In SoCC, 2013.

[3] Amazon Web Services. http://aws.amazon.com/.

[4] Microsoft Azure. http://azure.microsoft.com/.

[5] A. Cockcroft. Benchmarking high performance I/O with SSD for Cassandra on AWS. http://techblog.netflix.com/ 2012/07/benchmarking-high-performance-io-wit h.html/.

[6] T. Condie, N. Conway, P. Alvaro, J. M. Hellerstein, K. Elmeleegy, and R. Sears. MapReduce online. In NSDI, 2010.

[7] J. Dean and L. A. Barroso. The tail at scale. CACM, 56(2), Feb. 2013.

[8] A. D. Ferguson, P. Bodik, S. Kandula, E. Boutin, and R. Fonseca. Jockey: Guaranteed job latency in data parallel clusters. In EuroSys, 2012.

[9] Google Cloud Platform. http://cloud.google.com/.

[10] D. Ghoshal, R. S. Canon, and L. Ramakrishnan. I/O performance of virtualized cloud environments. In DataCloud-SC, 2011.

[11] Google Compute Engine. http://cloud.google.com/comp ute/.

[12] T. Graves. GraySort and MinuteSort at Yahoo on Hadoop 0.23. http://sortbenchmark.org/Yahoo2013Sort.pdf.

[13] A. Greenberg, J. R. Hamilton, N. Jain, S. Kandula, C. Kim, P. Lahiri, D. A. Maltz, P. Patel, and S. Sengupta. VL2: A scalable and flexible data center network. In SIGCOMM, 2009.

[14] H. Herodotou, F. Dong, and S. Babu. No one (cluster) size fits all: Automatic cluster sizing for data-intensive analytics. In SoCC, 2011.

[15] V. Jalaparti, H. Ballani, P. Costa, T. Karagiannis, and A. Rowstron. Bridging the tenant-provider gap in cloud services. In SoCC, 2012.

[16] D. Jiang. Indy Gray Sort and Indy Minute Sort. http:// sortbenchmark.org/BaiduSort2014.pdf.

[17] A. Li, X. Yang, S. Kandula, and M. Zhang. CloudCmp: Comparing public cloud providers. In IMC, 2010.

[18] Y. Low, J. Gonzalez, A. Kyrola, D. Bickson, C. Guestrin, and J. M. Hellerstein. Graphlab: A new framework for parallel machine learning. In $U A I, 2010$.

[19] G. Malewicz, M. H. Austern, A. J. Bik, J. C. Dehnert, I. Horn, N. Leiser, and G. Czajkowski. Pregel: a system for large-scale graph processing. In SIGMOD, 2010.

[20] P. Mehrotra, J. Djomehri, S. Heistand, R. Hood, H. Jin, A. Lazanoff, S. Saini, and R. Biswas. Performance evaluation of Amazon EC2 for NASA HPC applications. In ScienceCloud, 2012.

[21] M. Michael, J. E. Moreira, D. Shiloach, and R. W. Wisniewski. Scale-up $\mathrm{x}$ scale-out: A case study using Nutch/Lucene. In IPDPS, 2007.

[22] A. Rasmussen, M. Conley, R. Kapoor, V. T. Lam, G. Porter, and A. Vahdat. Themis: An I/O efficient MapReduce. In SoCC, 2012.

[23] A. Rasmussen, M. Conley, G. Porter, and A. Vahdat. TritonSort 2011. http://sortbenchmark.org/2011_06_tri tonsort.pdf.

[24] A. Rasmussen, G. Porter, M. Conley, H. V. Madhyastha, R. N. Mysore, A. Pucher, and A. Vahdat. TritonSort: A balanced large-scale sorting system. In NSDI, 2011.

[25] J. Schad, J. Dittrich, and J.-A. Quiané-Ruiz. Runtime measurements in the cloud: Observing, analyzing, and reducing variance. In $V L D B, 2010$.

[26] M. Sevilla, I. Nassi, K. Ioannidou, S. Brandt, and C. Maltzahn. SupMR: Circumventing disk and memory bandwidth bottlenecks for scale-up MapReduce. In LSPP, 2014.

[27] Sort Benchmark. http://sortbenchmark.org/.

[28] PCI-SIG Single Root IOV. http://www.pcisig.com/spec ifications/iov/single_root.

[29] SYSSTAT. http://sebastien.godard.pagesperso-ora nge.fr/.

[30] A. Verma, L. Cherkasova, and R. H. Campbell. ARIA: Automatic resource inference and allocation for MapReduce environments. In ICAC, 2011.

[31] vnStat - a network traffic monitor for Linux and BSD. ht tp://humdi.net/vnstat/.

[32] E. Walker. Benchmarking Amazon EC2 for high-performance scientific computing. LOGIN, 33(5):18-23, Oct. 2008.

[33] G. Wang and T. E. Ng. The impact of virtualization on network performance of Amazon EC2 data center. In INFOCOM, 2010.

[34] A. Wieder, P. Bhatotia, A. Post, and R. Rodrigues. Orchestrating the deployment of computations in the cloud with Conductor. In NSDI, 2012.

[35] R. Xin. Spark officially sets a new record in large-scale sorting. http://databricks.com/blog/2014/11/05/ spark-officially-sets-a-new-record-in-large-s cale-sorting.html.

[36] R. Xin. Spark the fastest open source engine for sorting a petabyte. http://databricks.com/blog/2014/10/10/ spark-petabyte-sort.html.

[37] R. Xin, P. Deyhim, A. Ghodsi, X. Meng, and M. Zaharia. GraySort on Apache Spark by Databricks. http://sortbe nchmark.org/ApacheSpark2014.pdf.

[38] Y. Xu, Z. Musgrave, B. Noble, and M. Bailey. Bobtail: Avoiding long tails in the cloud. In NSDI, 2013.

[39] Apache Hadoop. http://hadoop.apache.org/. 